\title{
COUPLING OF LO PHONONS TO EXCITONS IN GaN
}

\author{
A. Wysmolek, P. Łomiak, J.M. Baranowski, K. Pakula, \\ R. STĘPNIEWSKI, K.P. KORONA \\ Institute of Experimental Physics, Warsaw University \\ Hoża 69, 00-681 Warszawa, Poland \\ I. Grzegory, M. BoćKowski and S. PorowsKi \\ High Pressure Research Center, Polish Academy of Sciences \\ Sokołowska 29/37, 01-142 Warszawa, Poland
}

\begin{abstract}
The photoluminescence of homoepitaxial and heteroepitaxial GaN layers is reported. It is shown that the coupling between LO phonons and neutral accept or bound excitons is much stronger than the coupling between LO phonons and neutral donor bound excitons. In undoped homoepitaxial layer, in spite of that the no-phonon emission due to donor bound excitons is one order of magnitude stronger than the acceptor bound excitons emission, the predominant structure in the LO phonon replica of the excitonic spectrum is related to optical transitions involving acceptor bound excitons. Temperature studies showed that at higher temperature the LO phonon replica is related to free excitons.
\end{abstract}

PACS numbers: 78.55.Cr, 63.20.Mt, 71.55.Eq

Recent advance in homoepitaxial growth of GaN layers by metalorganic chemical vapour deposition (MOCVD) has demonstrated that high quality single crystal films can be grown on single crystal GaN substrates [1]. The quality of obtained layers gives the opportunity to obtain more detailed knowledge about optical properties of $\mathrm{GaN}$. In this paper we report on the observation of the LO phonon side-bands of the excitonic spectrum in homoepitaxial and heteroepitaxial MOCVD GaN layers.

The homoepitaxial wurtzite $\mathrm{GaN}$ were grown on single crystal GaN plates. The growth procedure of a high quality homoepitaxial GaN layers was described previously [1]. The heteroepitaxial GaN layers were grown on sapphire substrates in the same MOCVD system. The thickness of homoepitaxial and heteroepitaxial layers were of the order of $1 \mu \mathrm{m}$. The photoluminescence (PL) was excited by the $325 \mathrm{~nm}$ line of a He-Cd laser. Typically an incident power of $1 \mathrm{~mW}$ was used. The emission spectra were recorded with a spectral resolution of $0.05 \mathrm{meV}$ using SPEX 500M monochromator equipped with a photomultiplier. The calibration of the monochromator scale was performed on the basis of $\mathrm{Cd}$ atomic spectra 
visible in laser radiation. In wavelength to energy conversion the refractive index of air $n=1.00028$ was used [2]. Measurements were performed at broad range of temperature using helium cryostat.

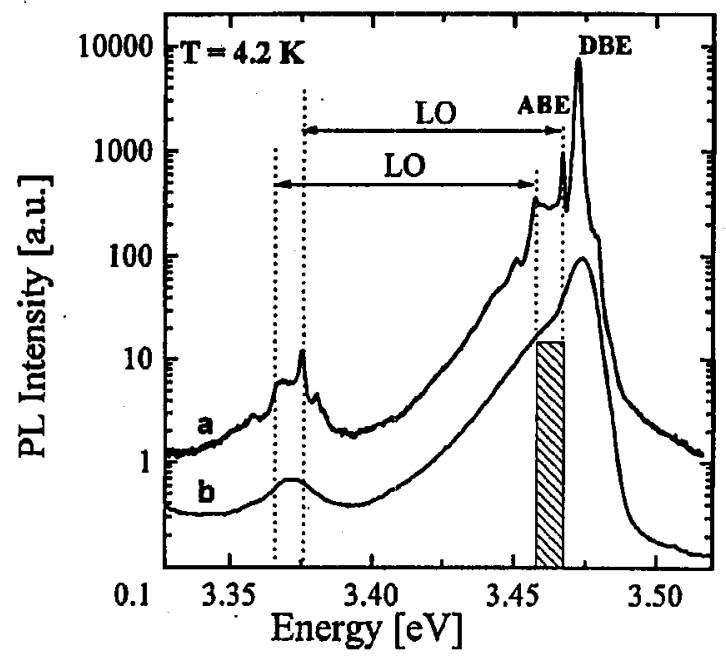

Fig. 1. Low temperature photoluminescence spectra of undoped $n$-type homoepitaxial (a) and heteroepitaxial (b) GaN layers. In the insert, the valence band structure of wurtzite $\mathrm{GaN}$ is shown.

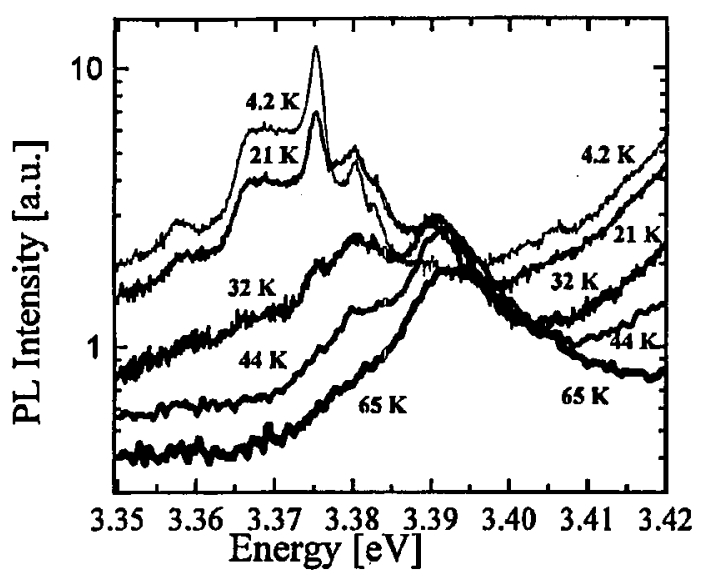

Fig. 2. The temperature changes of the LO phonon side-band observed in undoped $n$-type homoepitaxial layer.

Low temperature photoluminescence spectra of undoped $n$-type homoepitaxial and heteroepitaxial GaN layers are shown in Fig. 1. The homoepitaxial layer spectrum in the exciton region is dominated by emission connected with 
donor bound excitons (DBE) and acceptor bound excitons (ABE) which have been analysed before $[1,3]$. The LO phonon replica of the exciton region is also shown in Fig. 1. In spite of that the emission due to DBE is by one order of magnitude stronger than $\mathrm{ABE}$ emission, the predominant structure observed in the LO phonon side-band of the excitonic spectrum is related to the optical transitions in which ABEs are involved. Also the characteristic shape of the ABE is reproduced in the phonon replica. The intensity ratio of $\mathrm{LO}$ phonon to no-phonon $\mathrm{ABE}$ and DBE are substantially different; they are $1 \times 10^{-2}$ and $6 \times 10^{-4}$ respectively. The result that the LO phonons are so strongly coupled to ABE can be understood as the manifestation of the neutral acceptor-LO phonon bound state formation [4]. Such state can be formed in GaN because the ionization energy of shallow acceptor being close to $235 \mathrm{meV}$ [5] is much larger than the LO phonon energy (close to $92 \mathrm{meV}$ ). On the other hand, in the case of DBE the bound state with LO phonon will be much less probable because the energy of the LO phonon is larger than the donor binding energy estimated at $35 \mathrm{meV}$ [6]. Thus a neutral donor-LO phonon bound state would be degenerate with the free electron continuum and will spontaneously decay by ionization of the donor [7]. Consequently, the LO phonon replica related to DBE should have much lower intensity than the $\mathrm{LO}$ replica of $\mathrm{ABE}$, which is found experimentally.

The large intensity difference between LO phonon replicas related to DBE and $\mathrm{ABE}$ may lead to misinterpretation of the observed PL side-bands when the particular excitonic lines are not resolved. Such situation is shown in Fig. 1, where spectra. for homoepitaxial and heteroepitaxial layers are compared. In the het-

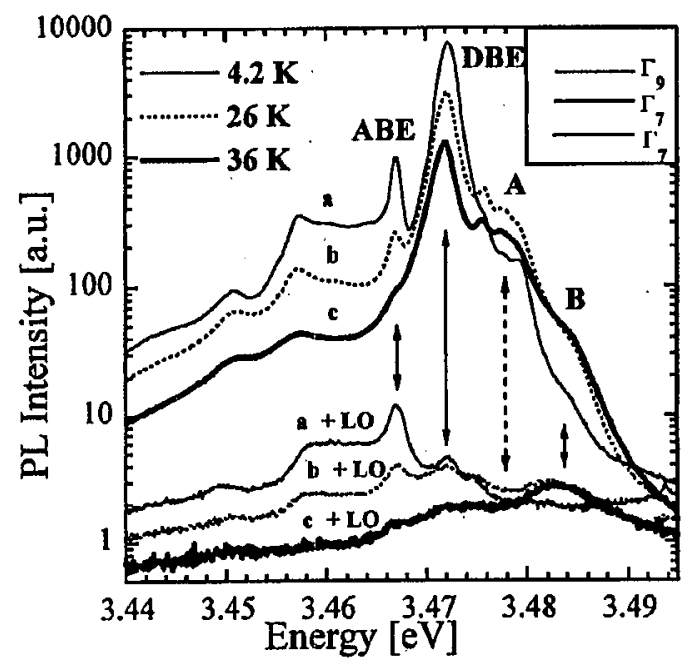

Fig. 3. The comparison of no-phonon excitonic luminescence (curves $a, b, c$ ) with LO phonon side-band (curves $a+\mathrm{LO}, b+\mathrm{LO}, c+\mathrm{LO}$ ). The phonon side-band was shifted by the LO phonon energy $(91.8 \mathrm{meV})$ to compare it with the main excitonic band. 
eroepitaxial layer the excitonic spectra are not resolved but the LO phonon replica is clearly seen. The comparison with the homoepitaxial spectrum allows to understand that LO phonons are only selectively coupled to the part of no-phonon spectrum which is related to ABE (shaded region shown in Fig. 1). Without such comparison one would be tempted to relate the observed side-band in terms of a new optical transition. The temperature changes of the LO phonon side-band are shown in Fig. 2. It is seen that the increase in temperature leads to the disappearing of first $\mathrm{ABE}$ and later DBE phonon replicas. This is in agreement with the temperature behaviour of the main exciton band. However, it is also seen that the increase in temperature leads to increase in emission at $3.39 \mathrm{eV}$. To identify this "hot" luminescence line we shifted the phonon side-band by the LO phonon energy $(91.8 \mathrm{meV})$ to compare it with the main excitonic band. This is shown in Fig. 3. It is seen that the "hot" line corresponds with free excitons emission. Simple inspection of the spectra suggests that this line corresponds to the phonon replica of the $B$ free exciton line. However, one should take into account that the shape of the phonon replica depends on temperature [8]. The analysis done along lines of the Permogorov paper shows that the phonon replica may be connected with free exciton $A$ as well.

This work was partially supported by the Committee for Scientific Research grant 7 T08 A 06110.

\section{References}

[1] K. Pakuła, A. Wysmołek, K.P. Korona, J.M. Baranowski, R. Stępniewski, I. Grzegory, M. Boćkowski, J. Jun, S. Krukowski, M. Wróblewski, S. Porowski, Solid State Commun. 97, 919 (1996).

[2] C.W. Allen, Astrophysical Quantities, University of London, The Althlone Press, London 1963, p. 119.

[3] R. Stępniewski, M. Potemski, A. Wysmołek, K. Pakuła, J.M. Baranowski, G. Martinez, I. Grzegory, M. Wróblewski, S. Porowski, to be presented at 23 Int. Conf. on Phys. of Semiconductors, 1996.

[4] P.J. Dean, D.D. Manchon, J.J. Hopfield, Phys. Rev. Lett. 25, 1027 (1970).

[5] A. Wysmolek, K.P. Korona, K. Pakuła, J.M. Baranowski, R. Stępniewski, I. Grzegory, M. Wróblewski, S. Porowski, to be presented at 23 Int. Conf. on Phys. of Semiconductors, 1996.

[6] B.K. Mayer, D. Volm, A. Graber, H.C. Alt, T. Detchprohm, A. Amano, I. Akasaki, Solid State Commun. 95, 597 (1995).

[7] C.H. Henry, J.J. Hopfield, Phys. Rev. B 6, 2233 (1972).

[8] S. Permogorov, in: Excitons, Eds. E.I. Rashba, M.D. Sturge, North-Holland, Amsterdam 1982, p. 177. 